\title{
Healthcare Monitoring System for Heart Diseases based on Internet of Things
}

\author{
Bowen Chen, Kateryna Hrabovska, Zihan Song and Oleksandr Lutsiuk \\ Linnaeus University
}

\begin{abstract}
In recent years, with the advancement of technology and medical information, smart home medical devices have become increasingly popular. However, medical care equipment for the elderly has not been widely mentioned. At present, it is still a problem for producing the devices that can comprehensively and deeply monitor the physical condition of the elderly. This article did the interview and Future Workshop methods to the target group, including medical staff and elderly, to collect and analyze information about their opinions in the healthcare monitoring system for heart diseases area, then designed a remote monitoring device based on the Internet of Things that monitors the user's (especially elderly) heart from both horizontal and vertical angles.
\end{abstract}

\section{Introduction}

In recent years, with the advancement of medical information, home medical equipment has become more and more popular among medical and health consumers. Simons(2008) and (Aragues et al., 2012) discussed the opportunities presented by the intersection of two different industries, family health and consumer electronics. At the same time, the popularity of smartphones and the development of Internet of Things technology (IoT) have brought about tremendous changes in the functions and forms of home medical devices. Spinsante and Gambi(2012) suggest that the middleware of the multimedia home platform allows personal health devices to interact with the TV. In the past, traditional home medical devices such as thermometers and weight scales were unable to provide users with comprehensive and continuous health management services due to their single function and lack of data storage capabilities. (Yan et al., 2010) and (Ivanov, Botvich and Balasubramaniam, 2012) have developed a wireless sensor network with specific functions. The new sensors in the network enable the acquired health data to be integrated with the remote gateway. Small portable and medical or health products with common consumer electronic functions greatly reduce the cost of health monitoring and management. The work carried out by Cornelius(2011) evaluates the configuration of equipment parameters in an open mobile health system to address the adaptation of the health equipment and smartphones carried.

Due to the rapid development and impact of information and communication technology (ICT), portable medical monitoring equipment faces severe service challenges in the 21 st century. (Viswanathan, Chen and Pompili, 2012) showed how it interfaces health monitoring systems with social networks. Through the early prevention of diseases, it can effectively prevent the growth of chronic diseases in the population. The cardiac reserve is an important aspect of cardiac function. Among the many methods for assessing cardiac reserve, the heart-heart-toheart relationship-based detection method has significant advantages of non-invasiveness and convenience. In this paper, our idea is a smart device that will be able to monitor heart rate, body temperature and temperature and humidity conditions in a room. If patient's results below the norm or above, caretaker or a nurse will receive the signal on the mobile application which will show patient's results, so responsible person can give needed attention to the patient.

Research in the field of personal health with autonomous health management and family health monitoring is important. Given the current inconsistency of different equipment manufacturers in domestic medical equipment, to develop medical equipment and information systems that can be interconnected, related applications and research are still in their infancy. In the application environment of personal health, the research on the information model of the medical information standard of the heart rate monitor is still in the blank. Domingo(2011) designed a context-aware architecture that uses IP-based multimedia subsystems to connect human sensing networks to social networks. From the perspective of IoT, (Bazzani et al., 2012) showed an IoT widget technology and served specific user-health scenarios. (Zhao, Wang and Nakahira, 2011) reviewed the opportunities and recommendations for medical applications in the IoT field. 


\section{Literature Review}

(Seena and Sudarshan, 2019) using the Raspberry Pi to develop an IoT platform, providing a health monitoring system for identifying human body conditions such as blood pressure, body temperature and heart rate on an IoT server through wireless network technology, in an emergency, a warning message/call will be automatically sent to the patient's caregiver, hospital, an ambulance if any strange data is found by the system. (Seena and Sudarshan, 2019) gives the concept of two platforms. A popular Raspberry Pi platform provides a complete Linux server at a very low price on a small platform, Raspberry allows interface services and mechanisms through a common I/O interface, through this combination, the proposed structure is more efficient, the Internet of Things connects these devices to provide an interactive life for humans.

(Malhi et al., 2012) developed Zigbee intelligent non-invasive wearable physiological parameter monitoring device, the human body's parameters can be monitored by this system through an electronic device worn on the wrist and the finger. The person will be monitored by using several sensors to measure different vital signs even in their own home, also there is an impact sensor has been used to detect if a person has a medical problem and alert the receiver connected to the computer, the device is battery powered and can be used outdoors and all in all is a very low-cost device which also suitable for athletes and babies (Malhi et al., 2012). (Roy and Gupta, 2014) introduced a short-range centralized health monitoring system that uses wireless ZigBee communication to collect ECG data for computer analysis, the ECG data was collected and compressed by a prototype of a patient data acquisition system based on ATmega16L microcontroller then the data will be wirelessly transmitted to a centralized station for remote terminal processing, also a software was developed to control patient modules and post-acquisition data analysis at the central station.

(Ahmed et al., 2018) proposed an automatic intelligent system based on Internet of Things, which can realize blood pressure, electrocardiogram and other functions, advanced database security, and include video conferencing system in the system to realize telemedicine for remote areas. The system automatically senses the patient's health and stores and displays the data via the Internet and informs the doctor about the condition. (S, P and P, 2019) proposes a project to continuously monitor different health parameters of patients through smartwatches. Smartwatches use different sensors to measure different health parameters, such as ECG, ppg and heart rate, and send this data to the cloud for further analysis, the data sent can be accessed from anywhere, also an emergency contact function is provided to the local surgeon or patient relation when the patient has an emergency, but the smartwatch must be connected to the smartphone via the android app.

A health monitoring system based on the concept of the Internet of Things was proposed in (Gutte and Vadali, 2018) and implemented on the Raspberry Pi. It is a remote health monitoring application which follows the basic MQTT protocol of the Internet of Things, the main purpose is to monitor the health of older people who are unable to visit the hospital regularly so that it also very easy to do primary health checks. In order to do the follow-up work, the patient's history is kept on the server (Gutte and Vadali, 2018).

Currently, there are many applications for patient health monitoring based on the Internet of Things. The usual approach is to monitor the data and transfer it to the server via an IoT platform or device, as we saw in the previous article. The main contribution is to monitor the patient's physical condition in real-time, to send the alert the doctor or related staffs promptly, and to provide more decision support for the doctor by collecting and analyzing the data. However, there are still some gaps that have not been mentioned yet. For examples, (1) There is no platform or device which can monitor all kinds of information mentioned from those articles. (2) No article clearly indicates that a specific patient's medical history can be performed, specifically analyzed. In another word, it can assume as the longitudinal analysis of a patient. There is also no function that can group all patients together and classify and manage according to the characteristics of the disease, that is, horizontal analysis and management of patients. (3) Some articles suggest that when abnormal data is detected, alarms will be issued to hospitals and guardians, but false alarms are not considered. Measures are not taken to reduce the chance of false alarms. For these gaps, the design mentioned in this article has the portability of the device and the intuitive characteristics of viewing information. The health care provider can obtain patient history information when targeting a specific patient. As for all patients, the health care provider has a simple visual interface that allows quick access to an overview of all the patients $\mathrm{s} / \mathrm{he}$ is responsible for.

\section{Case and context}

Heart rate refers to the number of times the human heart pulsates every minute. It is an important parameter that reflects whether the heart is working properly. According to the heart rate value, it can be judged whether a person has several common heart diseases such as tachycardia, and heart rate value is an important indicator for measuring physical labor intensity and mental labor intensity. Clinically, heart rate values can be obtained by ordinary electrocardiograph tests, but when measuring labor intensity, such electrocardiographs cannot be applied to the 
job site. The most effective way to assess the labor intensity of a job is to determine the heart rate value of the operator's work. Therefore, it is necessary to design a heart rate monitor that can be carried around, can record, display and store heart rate values for a long time, can communicate with the microcomputer and has the strong anti-drying ability.

By understanding the functional standards of existing heart rate monitors, the equipment envisioned in this paper should have the following features:

- Instantaneous heart rate value, heart rate variability value, average heart rate value and average heart rate variability can be measured.

- The heart rate value and heart rate variability stored in the data memory can be extracted and displayed on the liquid crystal display through the function keys.

- It can be controlled by function keys to send data in the data storage to the microcomputer, display heart-shaped electric waves on the computer, or save the data in the computer.

- If the measured heart rate value exceeds the normal heart rate value range, you can call the ambulance in time.

- The instrument also has several alternate function keys for further development of functions such as computer sample variance and display.

\section{Methodology and Design Process}

\section{Interview}

\begin{tabular}{|l|l|l|}
\hline \multicolumn{3}{|c|}{ Table 1. Interviewee Information } \\
\hline & Gender & Workplace \\
\hline Participants A & Female & Medical Student \\
\hline Participants B & Female & Cardiology Student \\
\hline
\end{tabular}

Table 1. Interviewee Information

The interview is to prepare some heart-related questions in advance, and the questions will show below. The interviews were in Chinese and Russian respectively, after which we listened to the recordings and translated them into English.

The questions we raised during the interview included:

1) How often elderly people approach you (as a medical specialist) with pain in the chest or thinking their chest/heart is hurting

2) If the elderly person has an actual heart problem, which non-heart related problems usually come with it (very related medical condition, symptom to track for better accuracy of detecting heart problems)

3) How often heart rate increased or cardiac pressure gained signifies heart diseases (maybe there are situations where the person feels not well and because of the nervous state its heart rate increases a little, so it's not actually because of heart disease)

4) what besides temperature and heart rate is necessary to track to detect critical heart conditions/heart diseases

5) According to the application's interface, what way of classifying would give you more convenient. (by gender, age or disease type?)

6) In addition to which accessibility features you would like, so that you can make better use of our products, for example, do you need the actual location of the patient to display

7) What data or records do you need at the time of switching the work?

8) Which of the features we currently offer are not needed or recommended for change. (Current basic functions: Heart rate and body temperature, ambient temperature, humidity.)

Based on the interview and the information we got from the participants we obtained the information about the organs and parameters in the human body that needs to be monitored in case of any abnormality, it is crucial in the matter of wellbeing because of a significant impact on other organs, blood vessels. 


\section{Future Workshop}

The future workshop is aims to enable and support the development of social fantasy that should lead to conflict resolutions that can be turned against the business-as-usual and the profit-seeking of the establishment (Jungk and Müller, 1987). Vidal (2006) mentioned the classic future workshop consists of five phases: the preparation phase, the critique phase, the fantasy phase, the implementation phase and the follow-up phase. It emphasizes collective work and collaborative learning through the interaction of the participants with the aim of learning how to build, sustain, and develop responsible participative communities (Vidal, 2006).

We invited four people to complete the process of the future workshop with us. Three elderly people over the age of 60 , one is a care worker. All three elderly people have different degrees of physical illness, and two of them have more serious heart problems. This female care worker often takes care of the elderly and understands the old people's living habits and psychological feelings. She provided a lot of constructive comments on the investigation and explained the role of functional equipment in the work of nursing workers, and encouraged us to practice it to a wider range of medical and living areas as soon as possible.

We arranged two meetings for the future workshop, and every group member attended. The first meeting was to complete the task assignment in the preparation phase. At the following time, we took the task with the Q\&A and thought about the project design. At the second meeting, we took colorful pens, paper boxes and stick-paper. Divide the white paper into four sections, which are design, functionality, experience with prototype and issues. Then we divided the whiteboard into three parts, namely critique phase, fantasy phase and implementation phase (presented in below figure 1). We added the thinking results of the corresponding stages in response to previous thinking. After completing the whiteboard marking process, the four of us began to discuss the latest findings of the project design and the corresponding feasible solutions. And also we will present the device mockup what we showed for people (Figure 2).

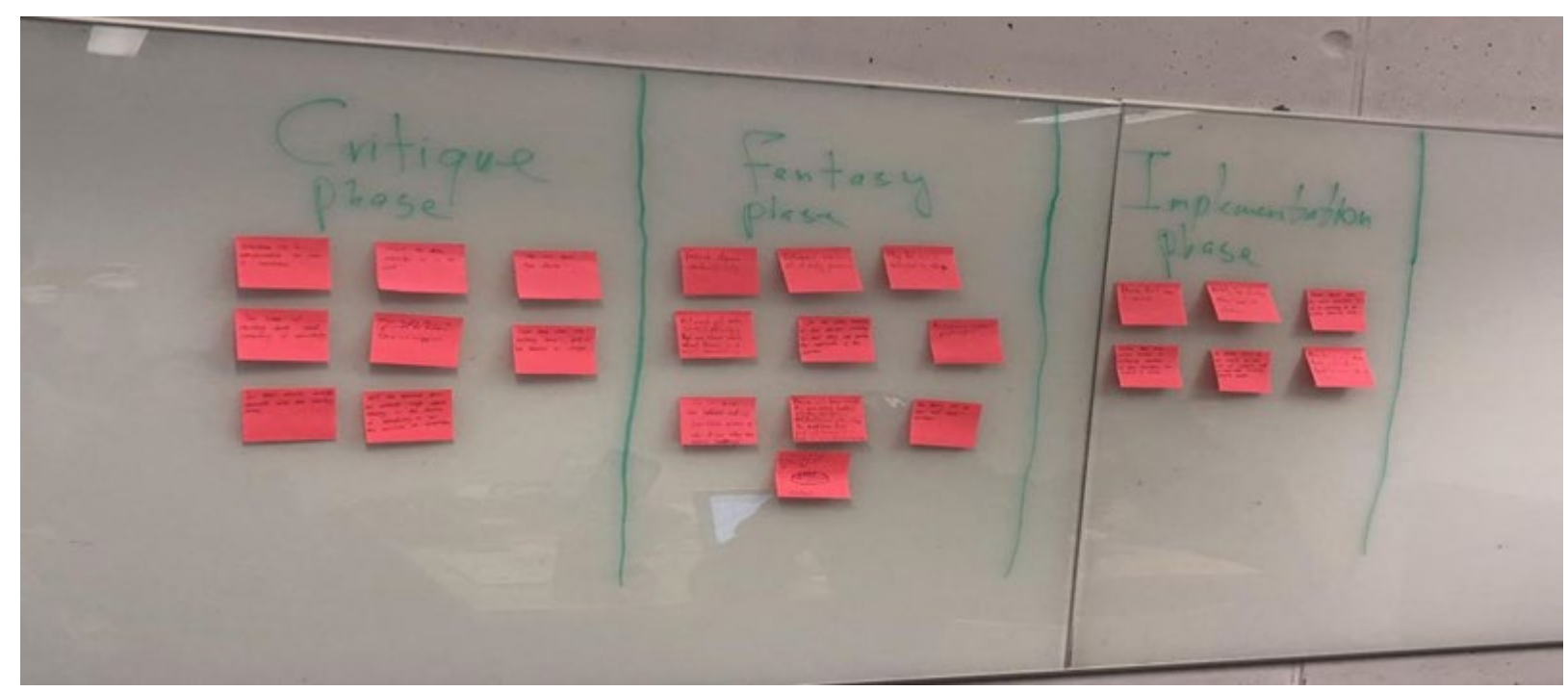

Figure 1. Future Workshop

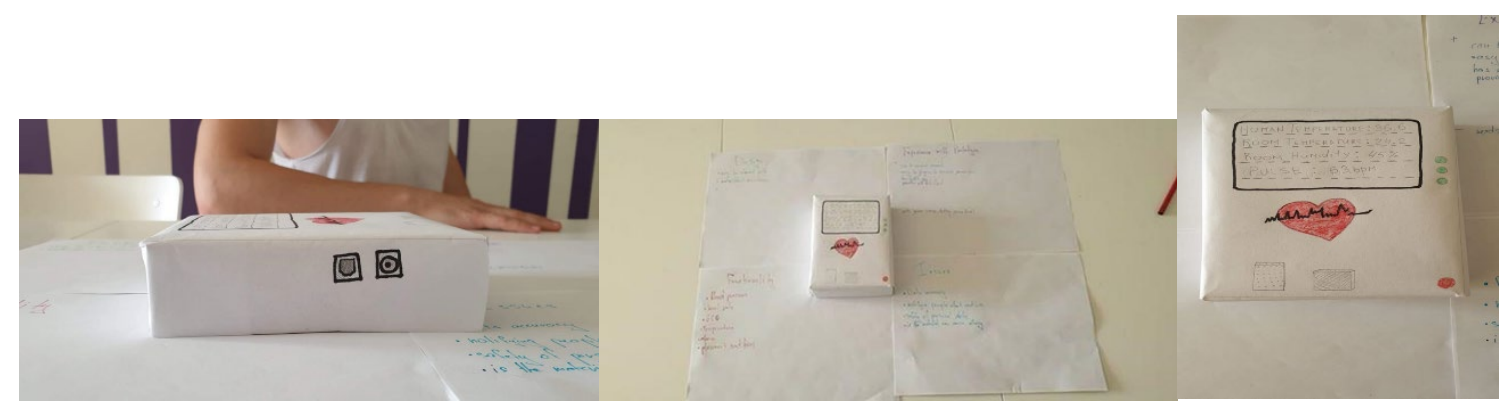

Figure 2. Device Mockup

\section{Analysis of data}

With the help of participatory design methods, it was possible for us to include in the work on this project endusers, which help us to design the device and analyze its functionality. Participatory design (PD) is an approach 
that allows all interested parties to actively participate in the creating process and helps to make sure that the results of the work are actually satisfy the needs of its users. This approach provides an opportunity to express the idea to the users and to get their point of view in order to create the most suitable solution for all parties, as well as it brings attention to some aspects of the project from the end-user point of view, this can be new solutions or critique of the incomprehensible elements of the design or too complex functionality (Huang, Lu and Sun, 2008).

The data we obtained from the interviews and future workshop was used to establish the importance of the project, requirements from the users, functionality that may be required from users, along with mockup design apprehensibility and users' concerns.

\section{Device Description}

This device supposed to be small enough and easily carried. As for the power, it may use the power bank or battery. This device will have a display screen so that users will be able to see the information directly. As for medical supervision part, the device will have a body temperature sensor; A sensor to measure room humidity and temperature, in order to monitoring and controlling the environment elements, This can not only help medical staffs to choose the specific etiological factor also can put patients in a comfortable environment. The device will also have a module to send the measurements to the caretaker's mobile device via Bluetooth.

For this project, we have 2 target groups - medical personnel/caretakers and elderly people.

While discussing the device functionality with medical personnel it has been discovered that elderly people tend to delay their visit to a medical facility in case of any health problem, so this device might be a good opportunity for their family or caretaker to keep track on their health. Based on the interviewees' medical experience elderly people willingly cooperate to be medically tested, so being connected to some device for health purposes won't be a problem, even if testing last for 24 hours. And important form factor that device is not big and heavy and can be carried around by elderly people. As for additional functionality, both medical specialists agreed on the necessity of implementing ECG in order to precisely monitor pulse and heart contraction. As for concerns both medical personnel pointed out data accuracy.

During the workshop with elderly people, we have established that people liked the idea of a device to keep track of health, that does not require any complex knowledge about technology and is easy to use. Also, they informed us that it is important that results information is visible to them and they don't have to set anything in the device to be able to use it. Also there were concerns regarding medical records privacy because the data will be sent to caretaker's phone people worried about information being intercepted and accessed by others, the support of the device - in case of malfunction how hard it will be to fix, the battery life - how long it will last and will they need a professional assistance in order to change it.

\section{Mobile Application}

After the device analyzed the human body and placement temperature and humidity the data sent to the mobile application installed on the caretaker's device. After discussing with the medical personnel what would be the most appropriate application solution, we established that the mobile application should have an easy and minimalistic interface - it won't take too much time from caretaker to check patient's condition, it should support multiple versions of the OS, because not everyone has up-to-date smart devices. And provide emergency information such as age, gender, medical records of previous health conditions, medicine allergies. All the information should be easily accessible and all measurements visible without additional actions, so caretaker will be able to check all at once and react if needed. The sound signal is also a useful feature for emergency cases.

\section{Handling Technical Issues}

When speech calls about modern devices for supervision upon old people, at once there is a row of questions about the storage of the personal data, problem, and complication of using a device. For providing confidentiality and reliable storage of data, medical data each of the users will be kept only on the device or user. It will provide to keep the calmness of users and not experience medical data for distribution.

So that during using this device there were no difficulties, we removed be what tuning for a device. Exactly due to such decision, be what user not depending on age or technical capabilities will be able without no problems to influence and use a device for own necessities. For management, it is necessary simply to connect a device to the telephone and open mobile edition, whereupon all data will be represented on the screen. 


\section{Prototype Design}

Design is an important part of the device, that is responsible for the usability and functionality of the device. Doing a circumspect design plan will help in creating a comfortable to use solution because creating a model first will provide an opportunity to perform an analysis before creating a product to elude problems in the actual software or hardware solution, it will become clear what elements should be changed, what problems should be fixed (McEwen and Cassimally, 2014).

The first thing to start doing our prototype was sketching. This step allows to put the abstract ideas on the paper and give it a shape. New ideas can be easily applied to the mockup. This step will help to visualize the external view of our device and will provide the better insight on what may be an issue and the possibilities to fix it (Mingzhu Li et al., 2010).

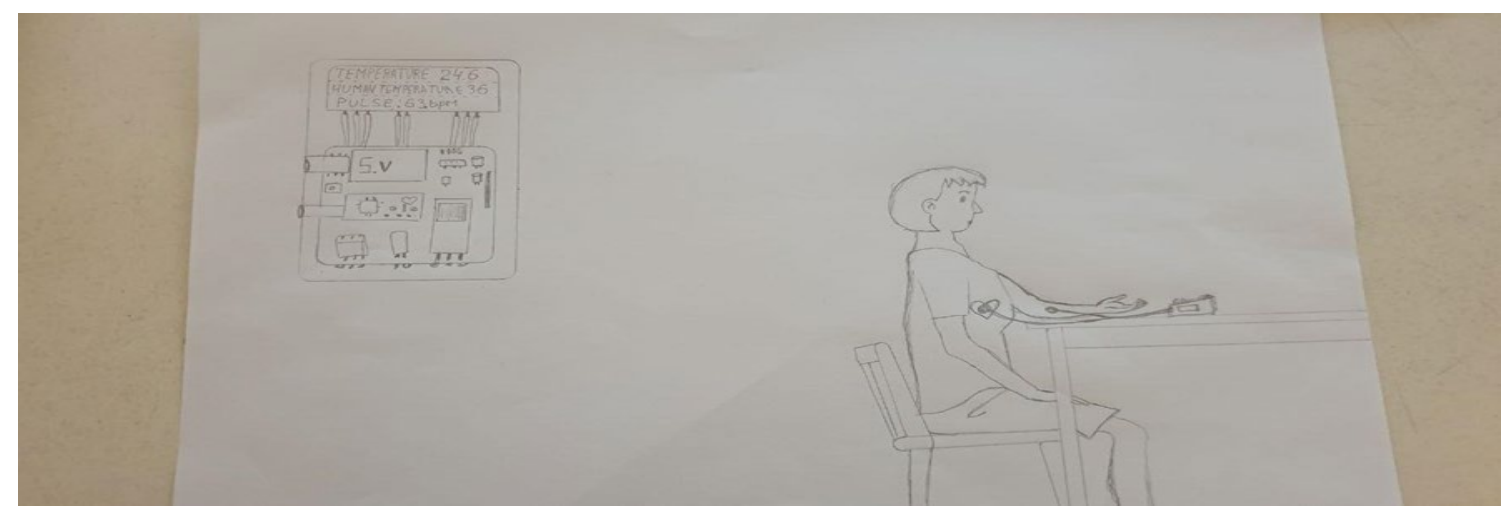

Figure 3. Sketch of Arduino Prototype

During the interview with 2 medical students and future workshop with elderly people we have established the following functional key points of the device:

- Measure human temperature

- Measure room temperature

- Measure room humidity

- Perform an ECG scan

- Measure heart rate

- Measure blood pressure

- Provide the user with their health data

- Provide responsible medical personnel/caretaker with the health data

- Notify the responsible medical personnel/caretaker in case of abnormal health changes

- Easy to read interface for both user parties

On the device's display, the patient will be able to see all health data results and the room's he is in temperature and humidity, this will help to maintain the comfortable conditions for patients.

The data on the display will be shown automatically, the patient doesn't need to set any configurations.

As for mobile application when medical personnel/caretaker party opens the app they welcomed with the patient's identifier, so when tapped on it their medical data will be visible to the responsible medical personnel/caretaker. The page with the information contains labelled fields for user's data, the actual results data from the sensors and the tab "History", that contains the user's (patient's) data for some time. Also, in case of any abnormal changes in patient's health the medical personnel/caretaker 's device will make a sound, which signifies there's something beyond usual and patient requires medical assistance. 


\section{App Prototype}

The process of designing application brought to us the view and understanding how it should look like and what functional items it should have. So, based on the results we got from interviews and future workshop we have developed the prototype of this mobile application. The application design has a login page, where the person who takes care of the patient-user can create and login an account to see the results data. Caretaker's account contains a list of patients. If you want to select a particular patient you may tap on their name and after the tap caretaker will see the window with the patient's data such as current state - the results from the device - in the "INFO" tab, "HISTORY" tab provides patient's results for some period of time and "MEDICAL RECORDS" tab provides medical information about pre-existing conditions and general information about patient, such as age, gender.

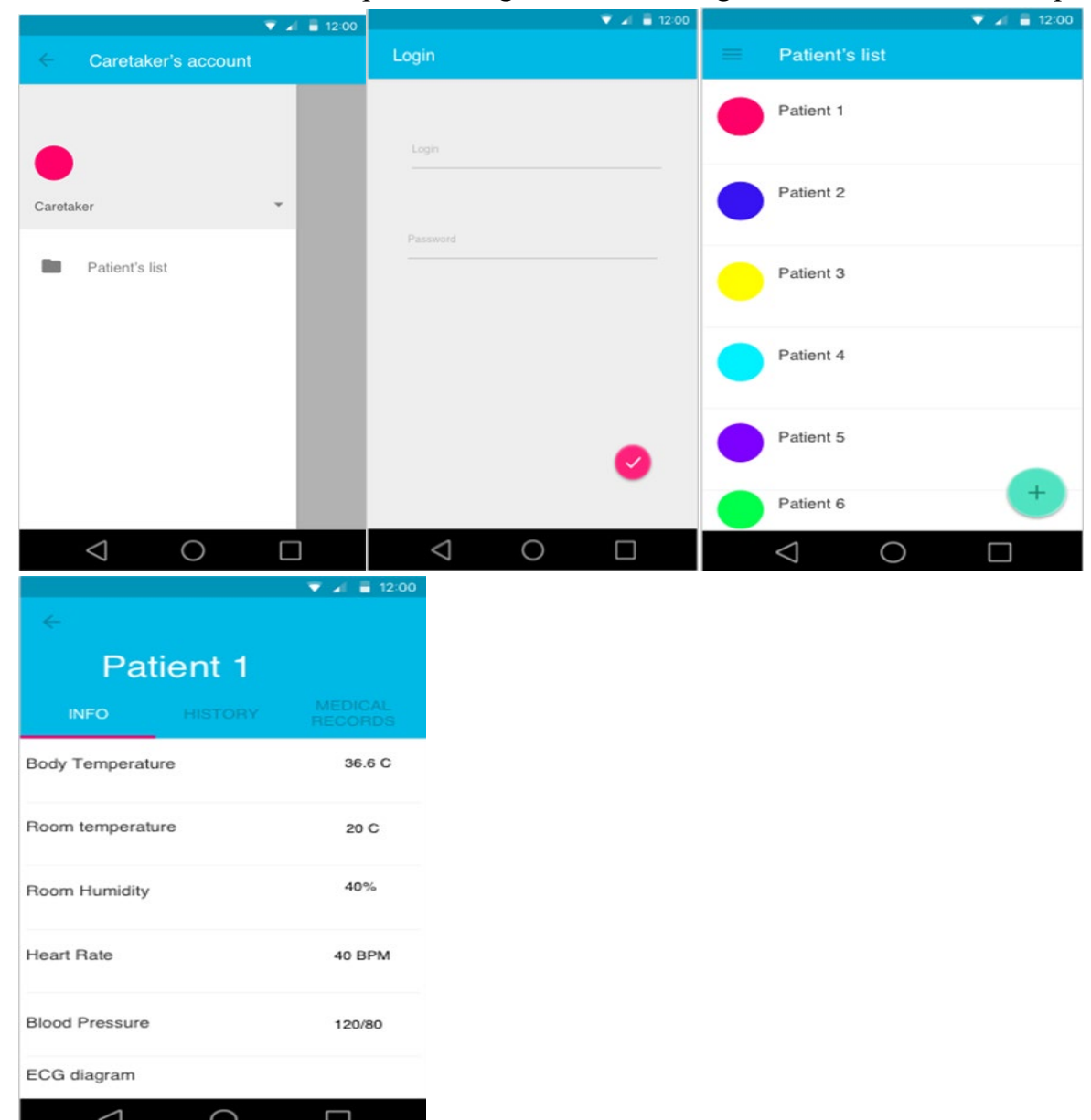

Figure 4. Mobile Application Sketch

\section{Arduino Prototype}

For prototyping the device itself, the Arduino platform has been chosen. Arduino is a computing platform that consists of a microcontroller - part that can be programmed, connected to the breadboard and provide "power" to the modules, plugged in the breadboard; the breadboard - part that provides "connection" to the various modules and microcontroller via special wiring; wires; modules that can be plugged into breadboard and provide necessary functionality. The Arduino prototypes can be dependent on the computer software they're running from or standalone - meaning that prototype only needs to be programmed and connected to the power source to function. Originally Arduino was meant to create designs and functional prototypes with some knowledge of programming and electronics (Galadima, 2014).

\section{Prototyping Functionality}

Arduino prototype for this project has been programmed with the use of Arduino IDE. In order to measure the temperature and humidity of the room, the special sensor should be connected to the breadboard, which in turn, 
connected to the microcontroller. In our case this sensor is DHT11. The sensor contains a humidity sensing component, an NTC sensor (thermistor) and IC. Humidity sensing component contains 2 electrodes and while humidity in the room changes the resistance between electrodes changes, this data passed to the IC that process it for the microcontroller. The changes in temperature are registered by a thermistor, where resistance changes with the variation in temperature. For measuring human temperature TMP36 sensor was used. This is a low voltage sensor, that generates an output result as a voltage that needs to be converted to Celsius or Fahrenheit. This sensor does not consist of the resistor, like the DHT11, instead, it uses the diodes - diode changes temperature which leads to voltage changes according to it. To send the data to the application on the mobile device HC-05 Bluetooth module was used. To make the modules work they need to be plugged in the breadboard with all the PINs being connected according to the circuit, and the breadboard needs to be connected to the microcontroller. After connecting PINs the microcontroller can be programmed with the usage of Arduino IDE and some libraries, which provides an opportunity to configure and read output from specific modules.

The results of the measurements are sent to the mobile application for the caretaker to see and displayed on the LCD monitor, connected to the breadboard, so that patient can also keep track on the health data.

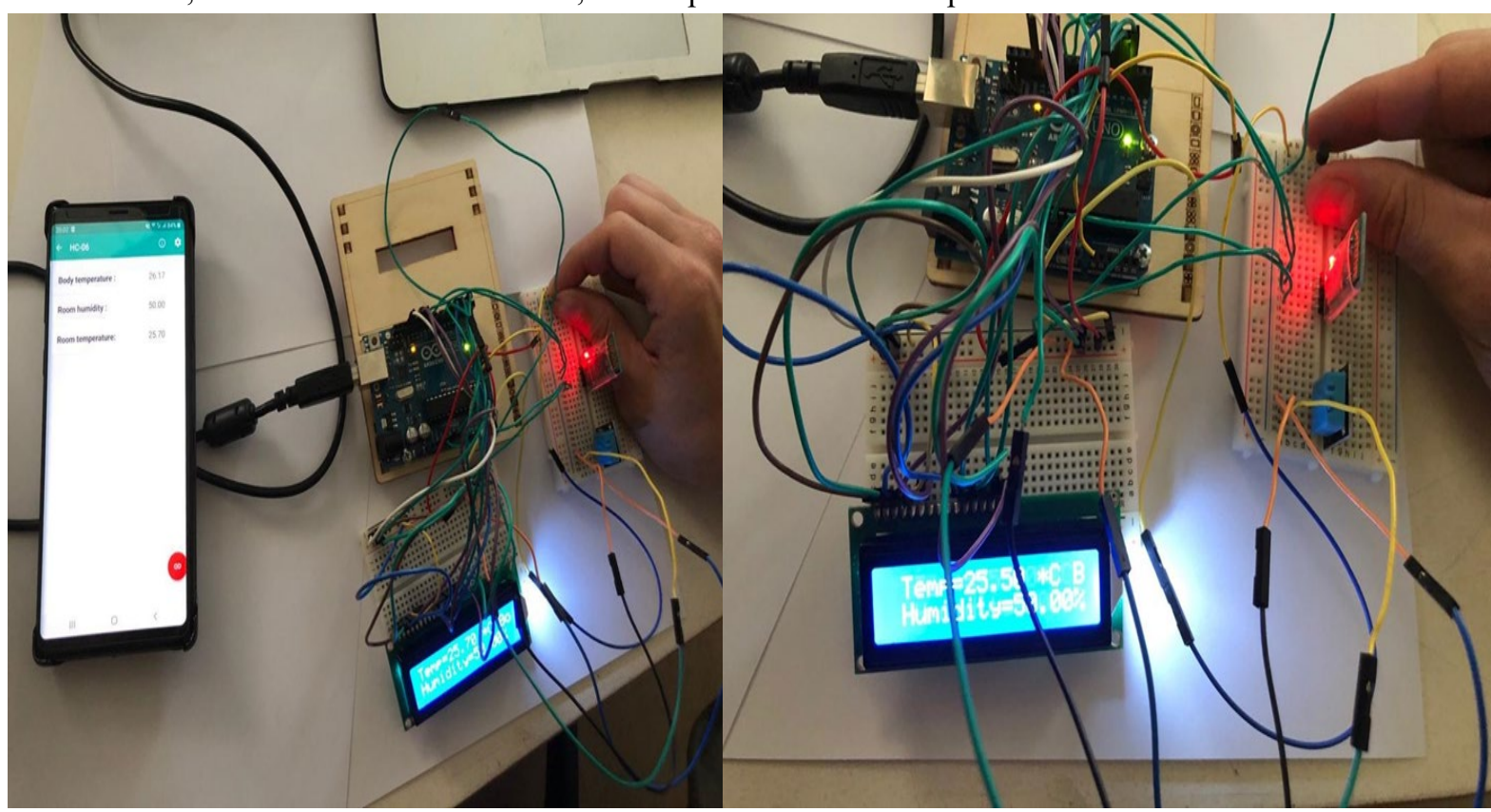

Figure 5. Arduino and Mobile Application Prototype of Healthcare Monitoring System

\section{Prototype Evaluation}

To evaluate the prototype we have involved the same group of people, that were involved in the future workshop and interview. According to the requirements, feedback from people and our vision of the device we have created a prototype with the use of Arduino and a mobile application for caretakers to review the data. To make value judgements about the prototype we have given the Arduino prototype to elderly people and inspect their interaction and reaction. Their main concern was the body temperature sensor being small and if someone has a tremor it will be a bit hard to interact with it. Some people expressed their concern in touching the module, if it is safe and won't give an electric shock to the person. And one person expressed concern about wires and modules being close together so it is possible to damage other parts of the device. Modules and wires problem can be solved by creating a 3D model of a case for such device, so modules and wires can be separated and hidden and each module will have a name, so there won't be any confusion. The electricity problem will be explained in the device instruction - modules are not dangerous and won't give an electric shock to the user. And the temperature sensor can be strapped to special clip and can be secured to the finger, so that tremor won't be a problem.

Medical personnel involved in mobile application design evaluation part expressed a positive opinion on the minimalistic design. 


\section{Discussion}

Analyzing the data, we can resume that take on the project idea and prototype was mainly positive. Both target groups welcomed the idea in a positive key, but also voiced their concerns regarding some aspects. Elderly people and medical personnel agreed that the device's form-factor is comfortable. On the other hand, it does not monitor all of the body, there are many health conditions that require specific sensors to detect. At the same time, based on the recommendations of medical staff and the elderly, we have determined the threshold of monitoring data, for example, normal heart rate is 60-100 times per minute, and so on. The data security issue was discussed and it is also concerning people. Also, it was discussed the device and application support - will it be updated and how hard it will be to fix a device in case of any malfunction. And while planning on creating a functional prototype we have decided to include in the realization the ECG module, which will monitor the heart and will help to diagnose heart diseases faster and accurately, but due to some implications, we haven't been able to receive this module. So, this part of functionality will be in the future work.

\section{Ethical Considerations}

Although the costs and benefits of new medical technologies have been widely discussed in recent years, much less attention has been paid to the entrance of such technologies into healthcare systems and the mechanisms that are or should be required for their acceptance. For instance, what kinds of studies are required for their approval? What kind of consent should be obtained from patients before a new technology is applied? These are questions that demand clear answers (McMahon et al,. 1987). It will not easily change As long as more advanced devices are produced, also undoubtedly will result in all sorts of potential damages and cause the growing commoditization of medicine. These are morally reprehensible. In addition, facing the overwhelming development of technology, we should be more and more cautious of its use, especially if it starts as promising and successful.

It is, therefore, necessary to take a look at these aspects from a different perspective and to discuss why modern technology has become a matter of philosophy and its "disciple" discipline: ethics. In the ethical sense, the realization of medical technology is based on the principles that everything should be done from the point of view of what is valuable, right and correct (Toader and Damir, 2014). Consequently, the significant impact of medical technology is expected to preserve human rights in healthcare, especially for people with disabilities and older people, where the need for rehabilitation is fast due to demographic surge and ageing the population all over the world. But the contribution of ethics in evaluating medical technologies should go beyond questions arising from their introduction into clinical practice; Ethics should focus on approaches that would allow determining the problems generating the moral effects of technologies, by dividing technical and non-technical issues related to medical practice (Toader, 2013). However, ethics due to the complexity of work in this field can turn the full potential of advanced medical technologies into profit by increasing their value (Braunack-Mayer, 2006).

As is usual in most ethical dilemmas, there are no precise answers to ethical problems; all cases can be viewed from multiple perspectives. Great healthcare design is born out of a strong collaborative relationship among healthcare representatives and the design team, a collaborative relationship which is built on trust, founded on integrity, and sealed infidelity and veracity. The healthcare industry and healthcare design field must have confidence that the scholarly findings and evidence in their fields of knowledge are being disseminated with the help of editors who are fair and have a strong commitment to the peer review process and a sense of responsibility and accountability. The developing science depends on the fidelity and veracity of its academic and practice researchers, healthcare-decision makers and designers.

\section{Conclusion and Future Work}

The main purpose of this project is to develop an IoT-based heart-health-monitoring prototype for the elderly. To achieve this, we used the interview and the method of the future workshop in PD. We let the participants participate in the actual operation of the project. This method helps us to truly integrate our target group into the whole project. On this basis, after data analysis, we carried out prototyping, including its functions and application interface, as well as evaluating models and taking different countermeasures. So in general, our project is based on the principle of paying attention to the heart health of the elderly and designing the prototypes and functions of the users (health care staff and the elderly) as much as possible and finally produced considerable results. As for the future work for this project, there is still something that can be improved and requires changes in the future. For example, we should make a case for the device, so all sensitive parts will be protected from external exposure. It is also possible to add more modules to extend functionality for heart-related issues as well as other diseases. And ECG functionality also moved to future work because we were not able to receive necessary parts to implement it. Also using a server for storing data might be a good solution because the amount of data will only increase with this device growing in demand. So we have to consider to improve data storage aspect too. All in all, this equipment 
will monitor heart and body temperature, room temperature and many other things which can help elderly living independently, only to see a doctor in a serious problem.

\section{References}

Ahmed, Z., Mortuza, M., Uddin, M., Kabir, M., Mahiuddin, M. and Hoque, M. 2018. "Internet of Things Based Patient Health Monitoring System Using Wearable Biomedical Device." 2018 International Conference on Innovation in Engineering and Technology (ICIET).

Aragues, A., Martínez, I., Del Valle, P., Muñoz, P., Escayola, J., \& Trigo, J. D. 2012. "Trends in entertainment, home automation and e-health: Toward cross-domain integration." IEEE Communications Magazine, 50(6), 160-167. https://doi.org/10.1109/MCOM.2012.6211501

Bazzani, M., Conzon, D., Scalera, A., Spirito, M. A., \& Trainito, C. I. 2012. "Enabling the IoT paradigm in ehealth solutions through the VIRTUS middleware." In 2012 IEEE 11th International Conference on Trust, Security and Privacy in Computing and Communications (pp. 1954-1959). IEEE. https://doi.org/10.1109/TrustCom.2012.144.

Braunack-Mayer, A. 2006. "Ethics and health technology assessment: Handmaiden and/or critic?" International Journal of Technology Assessment in Health Care, 22(3), pp.307-312. https://doi.org/10.1017/S0266462306051191

Cornelius, C., \& Kotz, D. 2011. "Recognizing whether sensors are on the same body." In International Conference on Pervasive Computing (pp. 332-349). Springer, Berlin, Heidelberg.

Domingo, M. C. 2011. "”A context-aware service architecture for the integration of body sensor networks and social networks through the IP multimedia subsystem. IEEE Communications Magazine, 49(1), 102-108. https://doi.org/10.1109/MCOM.2011.5681022

Galadima, A. 2014. “Arduino as a learning tool.” 2014 11th International Conference on Electronics, Computer and Computation (ICECCO).

Gutte, A. and Vadali, R. 2018. "IoT Based Health Monitoring System Using Raspberry Pi." 2018 Fourth International Conference on Computing Communication Control and Automation (ICCUBEA). https://doi.org/10.1109/ICCUBEA.2018.8697681

Huang, Y., Lu, R. and Sun, S. 2008. "Designing a Cooperative Learning System: A Scenario and Participatory Design Based Approach." 2008 International Symposium on Computational Intelligence and Design. https://doi.org/10.1109/ISCID.2008.203

Ivanov, S., Botvich, D., \& Balasubramaniam, S. 2012. "Cooperative wireless sensor environments supporting body area networks." IEEE transactions on Consumer Electronics, 58(2), 284-292. https://doi.org/10.1109/TCE.2012.6227425

Jungk, R., \& Müllert, N. 1987. "Future Workshops: How to create desirable futures." Instution for Social Inventions, London, UK.

Malhi, K., Mukhopadhyay, S., Schnepper, J., Haefke, M. and Ewald, H. 2012. “A Zigbee-Based Wearable Physiological Parameters Monitoring System." IEEE Sensors Journal, 12(3), pp.423-430. https://doi.org/10.1109/JSEN.2010.2091719

McEwen, A. and Cassimally, H. 2014. "Designing the internet of things.” John Wiley and Sons, Ltd.

McMahon, L. F., D. Fleischer, and R. Levine 1987. "Emerging technology: Patient protection versus proliferation.” J Clin Gasfroenter 01 9:258-273.

Mingzhu Li, Zhangping Lu, Liqing Huang and Canqun He 2010. "Research on design sketch teaching from the view of creative thinking." 2010 IEEE 11th International Conference on Computer-Aided Industrial Design \& Conceptual Design 1. https://doi.org/10.1109/CAIDCD.2010.5681346

Roy, S. and Gupta, R. 2014. "Short range centralized cardiac health monitoring system based on ZigBee communication." 2014 IEEE Global Humanitarian Technology Conference - South Asia Satellite (GHTCSAS). https://doi.org/10.1109/GHTC-SAS.2014.6967579

S, A., P, S. and P, M. 2019. "Smart Health Monitoring System through IOT." 2019 International Conference on Communication and Signal Processing (ICCSP). https://doi.org/10.1109/ICCSP.2019.8697921

Seena Naik, K. and Sudarshan, E. 2019. "Smart healthcare monitoring system using raspberry Pi on IoT platform." ARPN Journal of Engineering and Applied Sciences, 14(4), pp872-876.

Simons, D. P. 2008. "Consumer electronics opportunities in remote and home healthcare." In 2008 Digest of Technical Papers-International Conference on Consumer Electronics (pp. 1-2). IEEE. https://doi.org/10.1109/ICCE.2008.4588118

Spinsante, S., \& Gambi, E. 2012. "Remote health monitoring by OSGi technology and digital TV integration." IEEE Transactions on Consumer Electronics, 58(4), 1434-1441. https://doi.org/10.1109/TCE.2012.6415017

Toader, E. 2013. "Technological interventions in medicine - Ethic considerations and guidelines for the medical practice." 2013 E-Health and Bioengineering Conference (EHB). https://doi.org/10.1109/EHB.2013.6707323 
Toader, E. and Damir, D. 2014. "Medical Responsibility as Moral and Ethical Foundation for the Professional Conduit." Procedia - Social and Behavioral Sciences, 149, pp.955-961. https://doi.org/10.1016/j.sbspro.2014.08.314

Vidal, R. V. V. 2006. "The Future workshop: democratic problem solving." Economic analysis working papers, 5(4), 21.

Viswanathan, H., Chen, B., \& Pompili, D. 2012. "Research challenges in computation, communication, and context awareness for ubiquitous healthcare." IEEE Communications Magazine, 50(5), 92-99. https://doi.org/10.1109/MCOM.2012.6194388

Yan, H., Huo, H., Xu, Y., \& Gidlund, M. 2010. "Wireless sensor network based E-health system implementation and experimental results." IEEE Transactions on Consumer Electronics, 56(4), 2288-2295. https://doi.org/10.1109/TCE.2010.5681102

Zhao, W., Wang, C., \& Nakahira, Y. 2011. Medical application on internet of things. https://doi.org/10.1049/cp.2011.0751 